\title{
INFLUÊNCIA DA INCLINAÇÃO DAS FIBRAS DA MADEIRA NA SUA RESISTÊNCIA AO CISALHAMENTO
}

\author{
INFLUENCE OF SLOPE OF GRAIN IN SHEAR STRENGTH OF WOOD
}

\author{
Edgar Vladimiro Mantilla Carrasco ${ }^{1}$ Judy Norka Rodo Mantilla ${ }^{2}$
}

\begin{abstract}
RESUMO
As tensões de cisalhamento em função da inclinação das fibras da madeira foram determinadas por meio de ensaios experimentais. Os corpos de prova foram obtidos de 12 vigas de madeira Eucalyptus saligna, seguindo as prescrições da NBR 7190 (1979). Os mesmos foram confeccionados com inclinação variável das fibras $\left(0^{\circ}, 15^{\circ}, 30^{\circ}, 45^{\circ}, 60^{\circ}, 75^{\circ}, 90^{\circ}\right)$. Através de análise estatística foi possível estimar os parâmetros de variabilidade das tensões de cisalhamento em função da inclinação das fibras da madeira. Essas tensões foram corrigidas para a umidade padrão de $12 \%$. Foi possível definir as equações empíricas que definem melhor este fenômeno, por meio de uma análise estatística robusta do conjunto de dados. Das equações propostas na literatura por Hankinson, Karlsen, DIN 1052 e Keylwerth, a formulação prescrita pela DIN 1052 (2007), com as devidas modificações, apresenta melhor adequação aos resultados experimentais.
\end{abstract}

Palavras-chave: fratura mecânica; inclinação às fibras; eucalipto.

\begin{abstract}
The shear stress as a function of the inclination of the wood fibers were determined through experimental tests. The test specimens were obtained from a sample of wood beams 12 Eucalyptus saligna, following the requirements of NBR 7190 . They were made with variable slope fiber $\left(0^{\circ}, 15^{\circ}, 30^{\circ}, 45^{\circ}, 60^{\circ}, 75^{\circ}\right.$, $90^{\circ}$ ). Through statistical analysis it was possible to estimate the parameters of variability of shear stress with the slope of the wood fibers. The shear stresses were corrected to standard moisture of $12 \%$. With robust statistical analysis of the data set, it was possible to define the empirical equations that best define this phenomenon. Equations proposed in the literature by Hankinson, Karlsen, DIN 1052 and Keylwerth, the formulation prescribed by DIN 1052, with necessary modifications, has a better fit to the experimental results.
\end{abstract}

Keywords: fracture mechanics; slope of fibers; eucalyptus.

\section{INTRODUÇÃO}

A madeira, como material altamente anisotrópico, tem suas características mecânicas influenciadas por diversos parâmetros, em particular pela inclinação de suas fibras. Já é fato comprovado que a resistência à compressão é influenciada pela inclinação das fibras (WANGAARD, 1950;
KOLLMANN; COTÊ, 1968; DINWOODIE, 1975; BODIG; JAYNE, 1982; GREKIN; SURINI, 2008), entretanto, a maior ou menor influência depende da espécie da madeira. Quanto à influência da inclinação das fibras na resistência ao cisalhamento são poucos os estudos e ainda assim há controvérsias. Dahl e Malo (2009), Ukyo e Masuda (2006) e Yamasaki e Sasaki $(2003,2004)$ afirmam que nos ensaios

1 Engenheiro Civil, Dr., Professor Titular do Departamento de Engenharia de Estruturas, Escola de Engenharia, Universidade Federal de Minas Gerais, Av. Antonio Carlos, 6627, Campus Pampulha, Bloco 1, $4^{\circ}$ Andar, Sala 4123, CEP 31270-901, Belo Horizonte (MG), Brasil. mantilla@dees.ufmg.br

2 Engenheira Civil, Dra ${ }^{\text {. }}$, Professora Assistente da Faculdade de Engenharia e Arquitetura, Universidade Fundação Mineira de Educação e Cultura, Rua Dom Modesto Augusto, 102, Apto. 200, Coração Eucarístico, CEP 30535-430, Belo Horizonte (MG), Brasil. judymantilla@hotmail.com 
convencionais para a determinação da resistência ao cisalhamento se supõe um comportamento elásticolinear, mas na realidade ocorre um comportamento não linear. Vários estudos foram realizados propondo diversos tipos de ensaios e de corpos de prova (DUMAIL; OLOFSSON; SALME, 2000; LIU, 1984; OLIVEIRA, 2004; YOSHIHARA; FURUSHIMA, 2003; YOSHIHARA; MATSUMOTO, 2005; HASSEL et al., 2009; YOSHIHARA et al., 2001). Dahl e Malo (2009) e Liu et al. (1999) Liu, Ross e Rammer (1996) indicam que o ensaio convencional de cisalhamento não é um ensaio puro devido à ocorrência de uma combinação de tensões normais e de cisalhamento, mesmo assim as normas nacionais como a NBR 7190 (ABNT, 1979) e internacionais, com as D14394 (AMERICAN SOCIETY FOR TESTING AND MATERIALS, 2007) e Eurocode5 (EUROPEAN COMMITTEE FOR STANDARDIZATION, 2005) continuam prescrevendo esse tipo de ensaio já que em projetos convencionais em madeira é suficiente essa consideração. Kollmann e Côté (1984), FOREST PRODUCTS LABORATORY (1999), Liu e Ross (1998), Logsdon e Jesus (2010) e Szücs (1992), no estudo da influência da direção de aplicação do esforço em relação às fibras da madeira na resistência ao cisalhamento, utilizaram princípios e teorias aplicadas à resistência à compressão. Alguns estabeleceram modelos teóricos, em geral considerando a madeira ortotrópica, outros estabeleceram modelos empíricos, baseados em resultados de ensaios.

Segundo Kollmann e Côté (1984), o modelo empírico proposto por Hankinson, equação 1 , com expoente $n=2$, tem sido o mais utilizado para obter a resistência à compressão inclinada às fibras. Já Karlsen et al. (1967) recomendam o modelo empírico apresentado na equação 2 , com expoente $n=3$. A norma DIN 1052 utiliza a equação 3 com o expoente $n=1$. Szücs (1992) adota a mesma expressão com o expoente $n=2$. Segundo Kollmann e Côté (1984), Keylwerth também desenvolveu uma expressão empírica com expoente $n=2$, mas para a variação do módulo de elasticidade em função da inclinação das fibras, que, para as resistências, assume a forma da equação 4. Os índices nas Equações 1, 2, 3 e 4 foram modificados para indicar que estas podem ser utilizadas para prever a resistência ao cisalhamento inclinada às fibras da madeira.

$$
\begin{gathered}
f_{v, \theta}=\frac{f_{v, 0} \times f_{v, 90}}{f_{v, 0} \times \sin (\theta)^{n}+f_{v, 90} \times \cos (\theta)^{n}} \\
f_{v, \theta}=\frac{f_{v, 0}}{1+\left(\frac{f_{v, 0}}{f_{v, 90}}+1\right) \times \sin (\theta)^{n}} \\
f_{v, \theta}=f_{v, 0}-\left(f_{v, 0}-f_{v, 90}\right) \times \sin (\theta)^{n} \\
f_{v, \theta}=\frac{f_{v, 0}}{\left(\cos (\theta)^{n}-\frac{f_{v, 0}}{f_{v, 90}} \times \sin (\theta)^{n}\right) \times \cos (2 \theta)+\frac{f_{v, 0}}{f_{v, 45}} \times \sin (2 \theta)^{n}}
\end{gathered}
$$

Sendo: $\mathrm{f}_{\mathrm{v}, \theta}=$ resistência ao cisalhamento em função da inclinação das fibras, $\mathrm{f}_{\mathrm{v}, 0}=$ resistência ao cisalhamento paralela às fibras e $\mathrm{f}_{\mathrm{v}, 90}=$ resistência ao cisalhamento normal às fibras.

Há controvérsia, entre os autores, sobre o valor do expoente (n) dos termos trigonométricos nas equações $1 \mathrm{a} 4$. Alguns autores indicam coeficientes diferentes, conforme o tipo de solicitação, outros indicam coeficientes diferentes para diferentes teores de umidade da madeira.

Logsdon e Jesus (2010) apresentaram um estudo sobre a variação da resistência ao cisalhamento em função da inclinação das fibras. Utilizaram a madeira peroba-mica (Aspidosmerma populifolium) e verificaram a adaptabilidade das quatro equações. Karlsen et al. (1967) apresentaram um gráfico, confeccionado a partir da equação 2 , com $n=3$, mostrando a variação das tensões de cisalhamento com o ângulo entre o esforço aplicado e a direção das fibras. Nota-se que o tema da variação da resistência com o ângulo entre o esforço aplicado e a direção das fibras ainda está em discussão. Não existe consenso entre os autores sobre qual é a melhor expressão a ser utilizada e nem mesmo sobre o valor do expoente em determinada expressão. Nesse sentido, os objetivos deste trabalho visam determinar experimentalmente o comportamento das tensões de cisalhamento da madeira de eucalipto saligna (Eucalyptus saligna) em função da inclinação de suas fibras, determinar a expressão empírica que melhor se adequa e verificar sua validade.

\section{MATERIAIS E MÉTODOS}

Para se conseguir uma amostragem representativa, as vigas de madeira foram escolhidas ao acaso, em pequenos lotes, em serrarias e em épocas diferentes. 
A madeira utilizada para os corpos de prova (CPs) foi eucalipto saligna (Eucalyptus saligna). Estas vigas são encontradas com espessuras máximas de $55 \mathrm{~mm}$ e larguras variadas.

Segundo Logsdon (1995), a variação da resistência ao cisalhamento paralela às fibras ao longo de uma peça de amescla (Trattinikia burserifolia) é tão pequena que pode ser desprezada. Assim, CPs semelhantes podem ser obtidos de uma mesma viga.

Os CPs foram confeccionados de 12 vigas selecionadas aleatoriamente. Foram confeccionados 96 CPs, sendo 84 CPs para ensaios de cisalhamento, mudando a direção das fibras com 12 CPs para cada inclinação e $12 \mathrm{CPs}$ para determinação do teor de umidade e massa específica, seguindo as recomendações da NBR 7190 (ABNT, 1979), ver Figura 1. As dimensões dos CPs para os ensaios de cisalhamento foram: $6,4 \mathrm{~cm}$ x $5 \mathrm{~cm}$ x $5 \mathrm{~cm}$, com um pequeno degrau, de $1,4 \mathrm{~cm} \times 2 \mathrm{~cm} \times 5 \mathrm{~cm}$, para fixação do dispositivo de ensaio, e uma seção cisalhante de $5 \mathrm{~cm} \times 5 \mathrm{~cm}$.
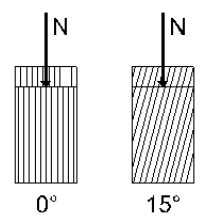
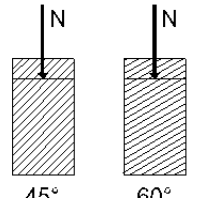

$60^{\circ}$

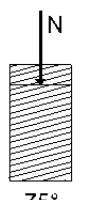

$75^{\circ}$

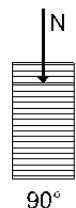

FIGURA 1: Corpos de prova com inclinação das fibras variável em relação à carga aplicada.

FIGURE 1: Test specimens with variable slope of the fibers in relation to the applied load.

Os ensaios de cisalhamento foram realizados em uma máquina universal de ensaios, com capacidade de $300 \mathrm{kN}$. O carregamento aplicado foi monotônico com velocidade de 2,5 MPa por minuto até alcançar a ruptura do CP. Após o término de cada ensaio de cisalhamento foram determinados os teores de umidade e densidade para correção da resistência a $12 \%$ de umidade. Para isso foi utilizada a equação 5 indicada pela NBR 7190 (ABNT, 1979).

$$
f_{v, \theta, 12}=f_{v, \theta, U \%}\left(1+\frac{2 \times(U \%-12)}{100}\right)
$$

Sendo: $\mathrm{f}_{\mathrm{v}, \theta, 12}=$ resistência ao cisalhamento inclinado de um ângulo $\theta$, em relação às fibras da madeira, no teor de umidade de referência de $12 \%$; $\mathrm{f}_{\mathrm{v}, \theta, \mathrm{U} \%}=$ resistência ao cisalhamento inclinado de um ângulo $\theta$, em relação às fibras da madeira, no teor de umidade $\mathrm{U} \%$, no instante do ensaio.

\section{RESULTADOS E DISCUSSÃO}

A umidade da madeira variou de $11,44 \%$ a $13,95 \%$ e a massa específica aparente de $690 \mathrm{~kg} / \mathrm{m}^{3} \mathrm{a}$ $860 \mathrm{~kg} / \mathrm{m}^{3}$. Na Tabela 1 apresentam-se os resultados das tensões de cisalhamento na ruptura e da umidade da madeira. Essas tensões já estão corrigidas para o teor de umidade de referência de $12 \%$.

Observou-se ruptura brusca e instantânea dos CPs, configurando-se uma ruptura frágil.

Na Figura 2 é apresentado o gráfico da tensão de cisalhamento em função da inclinação das fibras, tanto os valores experimentais (incluindo o intervalo de confiança), como as 4 equações com o expoente sugerido pelos respectivos autores. Notase que os gráficos com as equações de Karlsen e Keylwerth estão fora do intervalo de confiança para as inclinações de $15^{\circ}$ e $30^{\circ}$. As outras duas equações, Hankinson e DIN-1052, não estão representando satisfatoriamente os resultados experimentais, já que a curvas, destas equações, em algumas inclinações, estão nos extremos dos intervalos de confiança. Isso indica que os expoentes " $n$ " das equações poderiam ser diferentes dos sugeridos pelos autores.

Admitindo que os resultados experimentais tenham uma distribuição normal, como mostra o teste de Anderson-Darling com p-value de 0,493 e através da análise estatística da hipótese de diferença nula, para a comparação entre pares de indivíduos e grupos de indivíduos, foram comparados os valores experimentais com os equivalentes calculados pelas equações $1 \mathrm{a} 4$, utilizando a variação dos expoentes " $n$ " das funções trigonométricas. Assim, foi obtido o valor do expoente " $n$ " que apresenta melhor ajuste para cada equação. Na Tabela 2 é apresentada a relação dos resultados, dos testes de diferença nula, obtidos com a aplicação do programa Minitab 16 e sua análise. Para determinar o melhor valor de " $n$ " foram verificados diversos valores até encontrar o menor " $t$ " e o maior " $p$ ". Para determinar o intervalo de variação de " $n$ " foi analisado o intervalo de confiança, mesmo que contenha o zero, ainda assim, a hipótese nula é aceita. Na coluna observações são apresentados os resultados destas análises.

Ao analisar a Tabela 2, observa-se que a expressão de Hankinson (equação 1) tem validade estatística desde que o expoente "n" esteja no intervalo $1,85 \leq \mathrm{n} \leq 2,10$. O expoente que fornece 
TABELA 1: Tensões de cisalhamento na ruptura, corrigidas para umidade padrão de 12\%, (MPa).

TABLE 1: Shear stress rupture, corrected to standard moisture content of $12 \%(\mathrm{MPa})$.

\begin{tabular}{cccccccc}
\hline Vigas & $0^{\circ}$ & $15^{\circ}$ & $30^{\circ}$ & $45^{\circ}$ & $60^{\circ}$ & $75^{\circ}$ & $90^{\circ}$ \\
\hline 1 & 15,65 & 12,87 & 12,25 & 13,28 & 10,09 & 9,06 & 8,44 \\
2 & 17,14 & 15,44 & 14,35 & 11,36 & 12,95 & 10,16 & 9,76 \\
3 & 18,68 & 16,95 & 14,19 & 11,84 & 11,64 & 11,74 & 12,56 \\
4 & 17,85 & 16,12 & 14,75 & 12,68 & 13,08 & 13,47 & 11,31 \\
5 & 14,29 & 9,53 & 9,84 & 12,28 & 11,43 & 7,94 & 7,94 \\
6 & 14,08 & 14,19 & 11,60 & 10,47 & 10,14 & 9,01 & 8,22 \\
7 & 12,64 & 12,04 & 10,95 & 9,63 & 8,43 & 7,34 & 6,50 \\
8 & 14,47 & 11,97 & 10,00 & 9,60 & 9,74 & 6,71 & 6,97 \\
9 & 15,87 & 15,35 & 13,40 & 15,35 & 9,23 & 10,93 & 9,49 \\
10 & 17,78 & 16,10 & 12,32 & 13,44 & 11,90 & 11,06 & 11,48 \\
11 & 15,19 & 12,98 & 11,97 & 11,08 & 9,76 & 8,85 & 8,30 \\
12 & 14,05 & 12,14 & 11,33 & 10,83 & 9,72 & 8,78 & 8,22 \\
\hline Média & 15,64 & 13,81 & 12,25 & 11,82 & 10,68 & 9,59 & 9,10 \\
\hline CV (\%) & 11.43 & 15.49 & 12.83 & 13.69 & 13.35 & 19.48 & 19.64 \\
\hline
\end{tabular}

Em que: $\mathrm{CV}=$ Coeficiente de variação

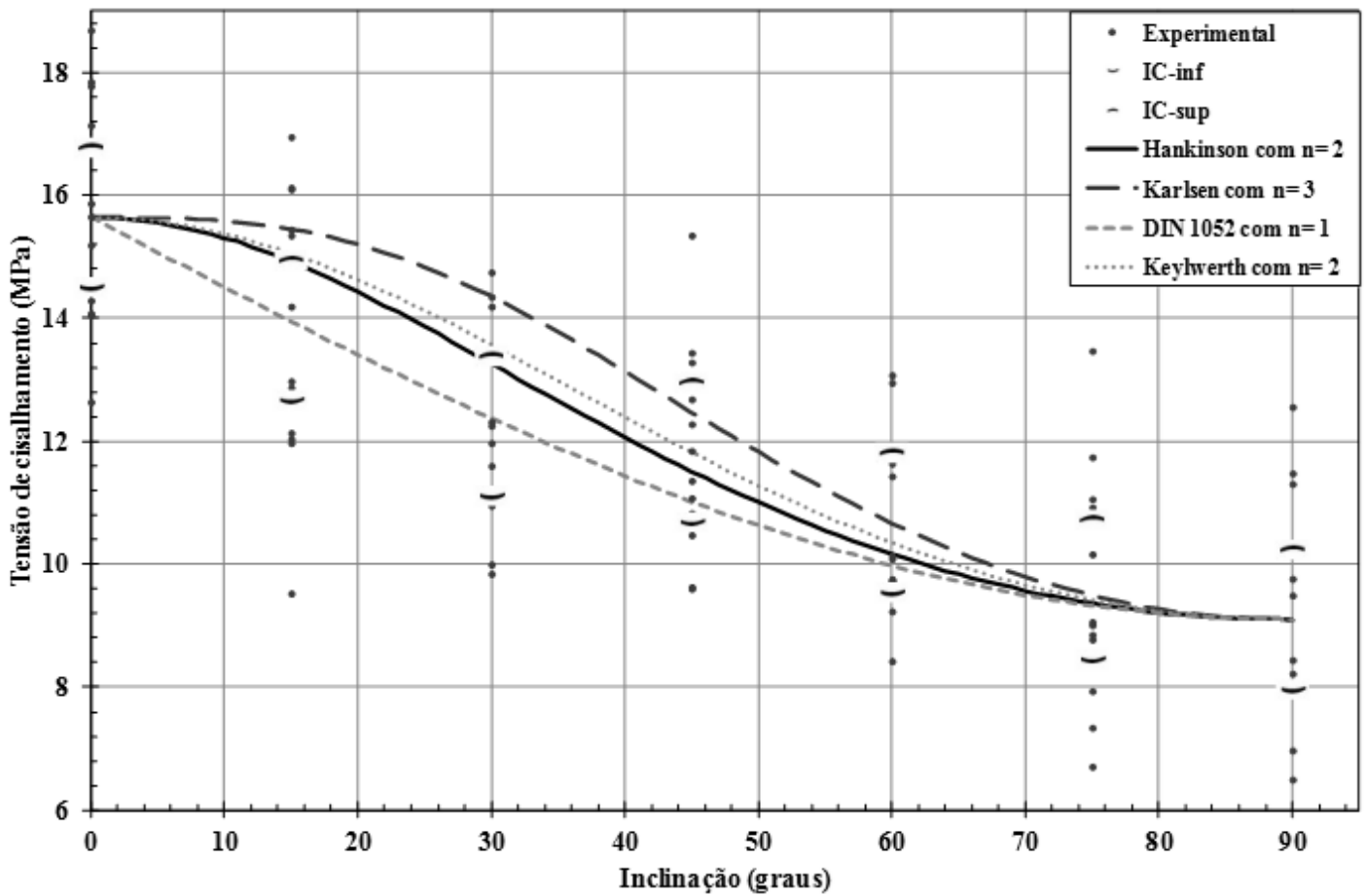

FIGURA 2: Tensão de cisalhamento $\mathrm{x}$ inclinação das fibras.

FIGURE 2: Shear stress versus inclination of the fibers.

melhor ajuste é $\mathrm{n}=1,93$. O valor tradicionalmente utilizado de $\mathrm{n}=2$, também apresentou validade estatística com uma significância razoável. Os valores encontrados por Logsdon e Jesus (2010), para a madeira peroba-mica (Aspidosmerma populifolium) são muito similares, $\mathrm{n}=2,05 \mathrm{e}$ intervalo de $1,88 \leq \mathrm{n} \leq 2,05$.

Para a expressão de Karlsen (equação 2) ter validade estatística o expoente "n" deve se encontrar no intervalo de $1,45 \leq n \leq 2,25$. O 
TABELA 2: Resultados dos testes de diferença nula entre os valores experimentais e as equações de 1 a 4 , variando o expoente " $n$ " (nível de significância de 95\%).

TABLE 2: Results of tests of zero difference between the experimental values and equations 1 to 4 , varying the exponent " $n$ " (significance level of 95\%).

\begin{tabular}{|c|c|c|c|c|c|c|}
\hline & & Difere & ça nula & entre os & resultados exper & imentais e as equações \\
\hline Eq. & $\mathrm{n}$ & $\begin{array}{l}\text { Difer. } \\
\text { média }\end{array}$ & $\mathrm{t}$ & $\mathrm{p}$ & $\begin{array}{c}\text { Intervalo de } \\
\text { confiança }\end{array}$ & Observações \\
\hline \multirow{6}{*}{ 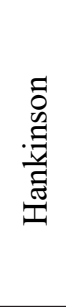 } & 1,80 & 0,272 & 2,12 & 0,037 & 0,$017 ; 0,526$ & Hipótese nula rejeitada \\
\hline & 1,85 & 0,169 & 1,32 & 0,192 & $-0,087 ; 0,424$ & Hipótese nula aceita \\
\hline & 1,93 & 0,005 & 0,04 & 0,967 & $-0,254 ; 0,265$ & Hipótese nula aceita - melhor ajuste curva \\
\hline & 2,00 & $-0,137$ & $-1,03$ & 0,308 & $-0,403 ; 0,129$ & Hipótese nula aceita \\
\hline & 2,10 & $-0,340$ & $-2,43$ & 0,017 & $-0,619 ; 0,062$ & Hipótese nula aceita \\
\hline & 2,15 & $-0,442$ & $-3,07$ & 0,003 & $-0,728 ;-0,155$ & Hipótese nula rejeitada \\
\hline \multirow{5}{*}{ 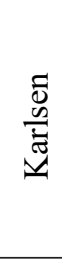 } & 1,40 & 0,261 & 2,08 & 0,041 & 0,$011 ; 0,521$ & Hipótese nula rejeitada \\
\hline & 1,45 & 0,223 & 1,77 & 0,081 & $-0,028 ; 0,474$ & Hipótese nula aceita \\
\hline & 1,77 & 0,001 & 0,01 & 0,993 & $-0,258 ; 0,260$ & Hipótese nula aceita - melhor ajuste curva \\
\hline & 2,30 & $-0,296$ & $-2,14$ & 0,036 & $-0,571 ;-0,020$ & Hipótese nula rejeitada \\
\hline & 3,00 & $-0,591$ & $-3,98$ & 0,000 & $-0,886 ; 0,148$ & Hipótese nula rejeitada (significante) \\
\hline \multirow{7}{*}{$\frac{n}{o}$} & 0,95 & 0,267 & 2,23 & 0,029 & 0,$029 ; 0,505$ & Hipótese nula rejeitada \\
\hline & 0,98 & 0,235 & 1,97 & 0,053 & $-0,003 ; 0,473$ & Hipótese nula aceita \\
\hline & 1,00 & 0,215 & 1,79 & 0,076 & $-0,023 ; 0,453$ & Hipótese nula aceita \\
\hline & 1,23 & 0,000 & $-0,00$ & 0,999 & $-0,243 ; 0,243$ & Hipótese nula aceita - melhor ajuste curva \\
\hline & 1,50 & $-0,212$ & $-1,68$ & 0,097 & $-0,465 ; 0,040$ & Hipótese nula aceita \\
\hline & 1,60 & $-0,282$ & $-2,19$ & 0,031 & $-0,538 ;-0,026$ & Hipótese nula rejeitada \\
\hline & 2,00 & $-0,522$ & 1,243 & 0,000 & $-0,794 ;-0,251$ & Hipótese nula rejeitada. (significante) \\
\hline \multirow{6}{*}{ 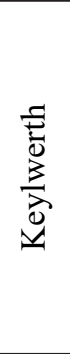 } & 0,80 & 0,103 & 0,59 & 0,557 & $-0,245 ; 0,451$ & $\begin{array}{l}\text { Hipótese nula aceita. Nova tendência para curva de melhor } \\
\text { ajuste }\end{array}$ \\
\hline & 1,00 & 0,129 & 0,81 & 0,422 & $-0,189 ; 0,447$ & Hipótese nula aceita \\
\hline & 1,51 & 0,001 & 0,00 & 0,996 & $-0,273 ; 0,274$ & Hipótese nula aceita - melhor ajuste curva \\
\hline & 1,90 & $-0,205$ & $-1,56$ & 0,123 & $-0,468 ; 0,057$ & Hipótese nula aceita \\
\hline & 1,99 & $-0,261$ & $-1,98$ & 0,052 & $-0,524 ; 0,002$ & Hipótese nula aceita \\
\hline & 2,00 & $-0,268$ & $-2,02$ & 0,046 & $-0,531 ;-0,004$ & Hipótese nula rejeitada \\
\hline
\end{tabular}

Em que: $\mathrm{t}=\mathrm{t}$ de student para teste de significância, $\mathrm{p}=\mathrm{p}$-value .

expoente que fornece melhor ajuste é $\mathrm{n}=1,77$. O valor tradicionalmente utilizado, recomendado por Karlsen et al. (1967) de $n=3$, não tem validade estatística e apresenta uma alta rejeição. Também o valor encontrado por Logsdon e Jesus. (2010) é diferente, $\mathrm{n}=2,13$, com pequena significância estatística. $\mathrm{O}$ estranho é que o intervalo de validade estatística, encontrado pelo autor citado, é muito similar ao encontrado neste trabalho, $1,71 \leq \mathrm{n}$ $\leq 2,26$. Isso pode ser devido ao tipo de madeira utilizado nos ensaios.

Já a expressão da DIN-1052 (equação 3) terá validade estatística desde que o expoente " $n$ " esteja no intervalo de $0,98 \leq \mathrm{n} \leq 1,50$. O expoente que permite melhor ajuste é $\mathrm{n}=1,23$. $\mathrm{O}$ valor utilizado por essa norma, $n=1$, encontra-se no intervalo, o que indica que tem validade estatística. Entretanto, esse modelo não tem validade estatística com $n=2$, proposto por Szücs (1992). O valor encontrado por Logsdon e Jesus (2010), n = 1,21, é muito próximo ao encontrado neste trabalho e tem alta significância estatística. Entretanto, o intervalo de validade estatística $1,16 \leq n \leq 1,27$, é diferente ao encontrado neste trabalho.

Para a expressão de Keylwerth (equação 4) não foi possível encontrar o limite inferior do 
intervalo de validade estatística para o expoente da expressão. Parece que existe na expressão uma singularidade que permite encontrar outro valor do expoente que forneça um ajuste do modelo altamente significante. O intervalo é "valor em aberto" $\leq \mathrm{n} \leq$ 1,99 e o expoente que proporciona melhor ajuste é $\mathrm{n}=1,51$. O valor tradicionalmente utilizado $\mathrm{n}=2$, recomendado por Kollmann e Côté (1984), não tem validade estatística. Também o valor encontrado por Logsdon e Jesus (2010) é diferente, $n=2,04$, sem significância estatística e o intervalo de validade estatística, $1,88 \leq \mathrm{n} \leq 2,22$ é diferente ao encontrado neste trabalho.

Com a finalidade de determinar qual equação e qual expoente "n" representa melhor os resultados experimentais e que tem maior significância estatística, foram traçados os gráficos das Figuras 3 e 4 . Esses gráficos foram obtidos da análise estatística das diferenças entre os valores experimentais e as equações 1 a 4 . Na Figura 4 é apresentado o histograma dos resíduos da diferença,
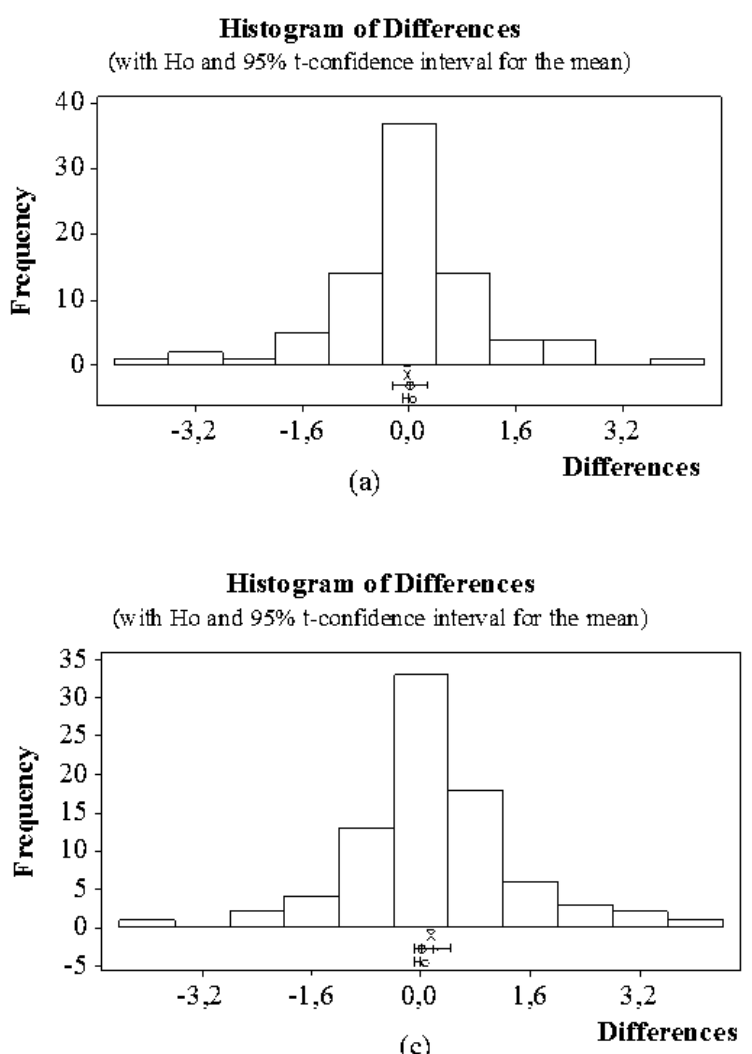

(c) no qual se nota que todos têm uma tendência de uma distribuição normal, contudo, a equação de Keylwerth (d) apresenta uma maior distorção dessa distribuição. Observa-se na Figura 5, com os gráficos boxplot dos resíduos da diferença, que o gráfico que representa a equação de Keylwerth (d) tem mais pontos outline e o intervalo de confiança está fora do box. Portanto, pode-se afirmar que apenas a equação de Keylwerth (equação 4) tem menos significância estatística que as outras equações.

Para uma avaliação mais robusta na escolha do melhor modelo que representasse os resultados experimentais foi feita uma análise One-way ANOVA. Nesse sentido, foram utilizados os métodos de Dunnett, Tukey e Fisher. Os três métodos indicaram que as equações de Hankinson, Karlsen e a DIN 1052 têm elevada significância e que a DIN 1052 apresenta melhor representatividade com elevada significância.

Finalmente, para poder visualizar a variação das tensões de cisalhamento em função

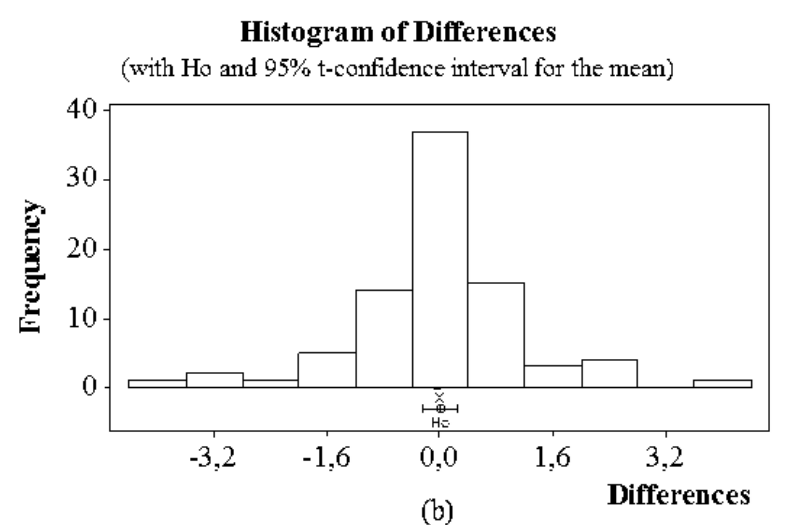

(b)

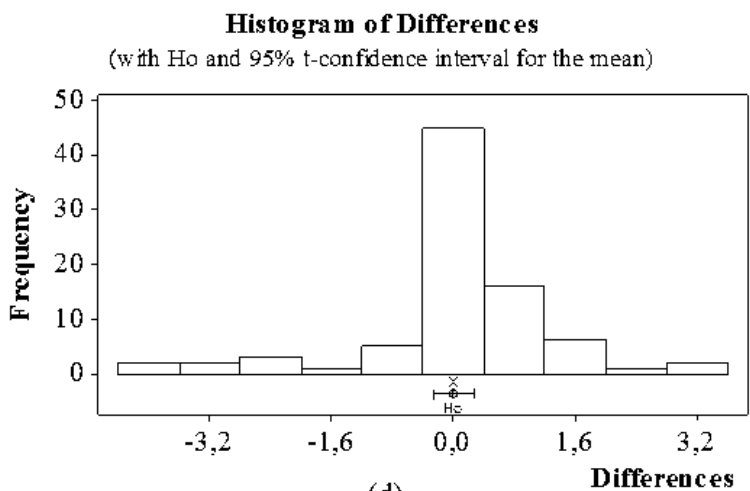

(d)

FIGURA 3: Histogramas das diferenças entre os resultados experimentais e das equações. a) - Hankinson $\operatorname{com} \mathrm{n}=1,93, \mathrm{~b})$ - Karlsen $\operatorname{com} \mathrm{n}=1,77, \mathrm{c})$ - DIN $1052 \operatorname{com} \mathrm{n}=1,23$ e d) - Keylwerth com $\mathrm{n}=1,51$.

FIGURE 3: Histograms of the differences between the experimental results and the equations. a) - Hankinson with $\mathrm{n}=1.93, \mathrm{~b}$ ) - Karlsen with $\mathrm{n}=1.77, \mathrm{c})$ - DIN 1052 with $\mathrm{n}=1.23 \mathrm{~d}$ ) - Keylwerth with $\mathrm{n}=1.51$. 
Boxplot of Differences

(with $\mathrm{Ho}$ and $95 \% \mathrm{t}$-confidence interval for the mean)

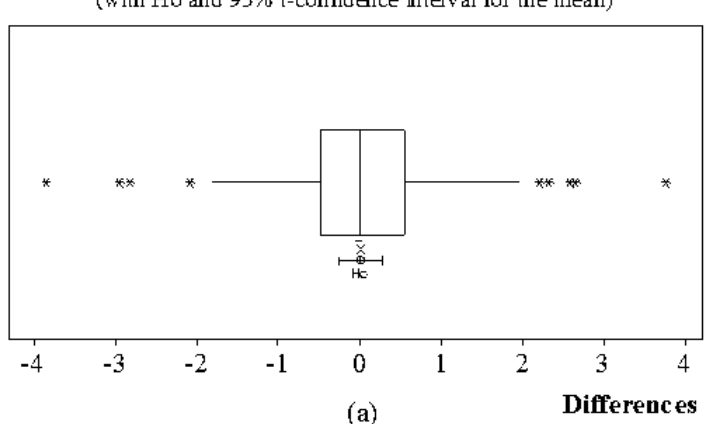

Boxplot of Differences

(with $\mathrm{Ho}$ and $95 \%$ t-confidence interval for the mean)

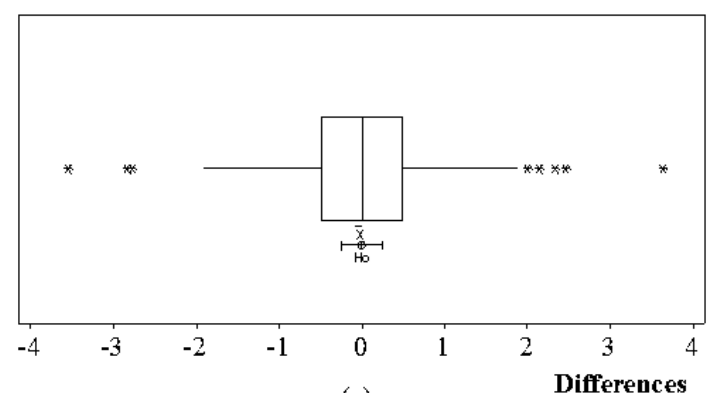

(c)
Boxplot of Differences

(with Ho and $95 \%$ t-confidence interval for the mean)

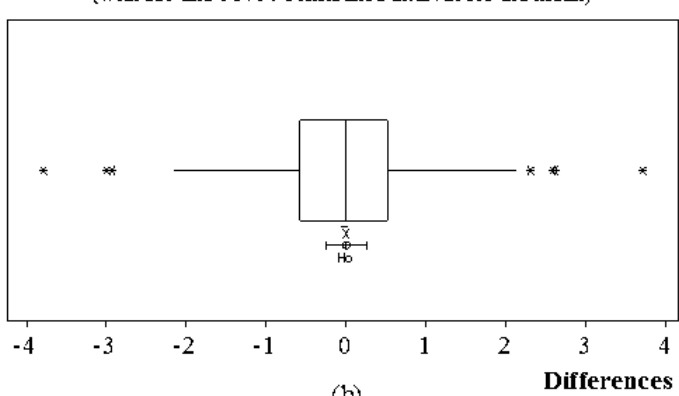

(b)

Boxplot das diferenças

(with $\mathrm{Ho}$ and $95 \% \mathrm{t}$-confidence interval for the mean)

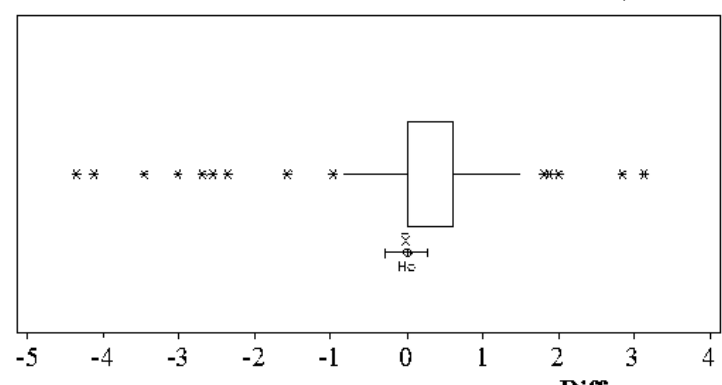

(d)

FIGURA 4: Boxplot das diferenças entre os resultados experimentais e das equações. a) - Hankinson com $\mathrm{n}=1,93, \mathrm{~b})$ - Karlsen com $\mathrm{n}=1,77, \mathrm{c})$ - DIN 1052 com $\mathrm{n}=1,23$ e d) - Keylwerth com $\mathrm{n}=1,51$.

FIGURE 4: Boxplot of the differences between the experimental results and the equations. a) - Hankinson with $\mathrm{n}=1.93, \mathrm{~b}$ ) - Karlsen with $\mathrm{n}=1.77, \mathrm{c})$ - DIN 1052 with $\mathrm{n}=1.23 \mathrm{~d}$ ) - Keylwerth with $\mathrm{n}=1.51$.

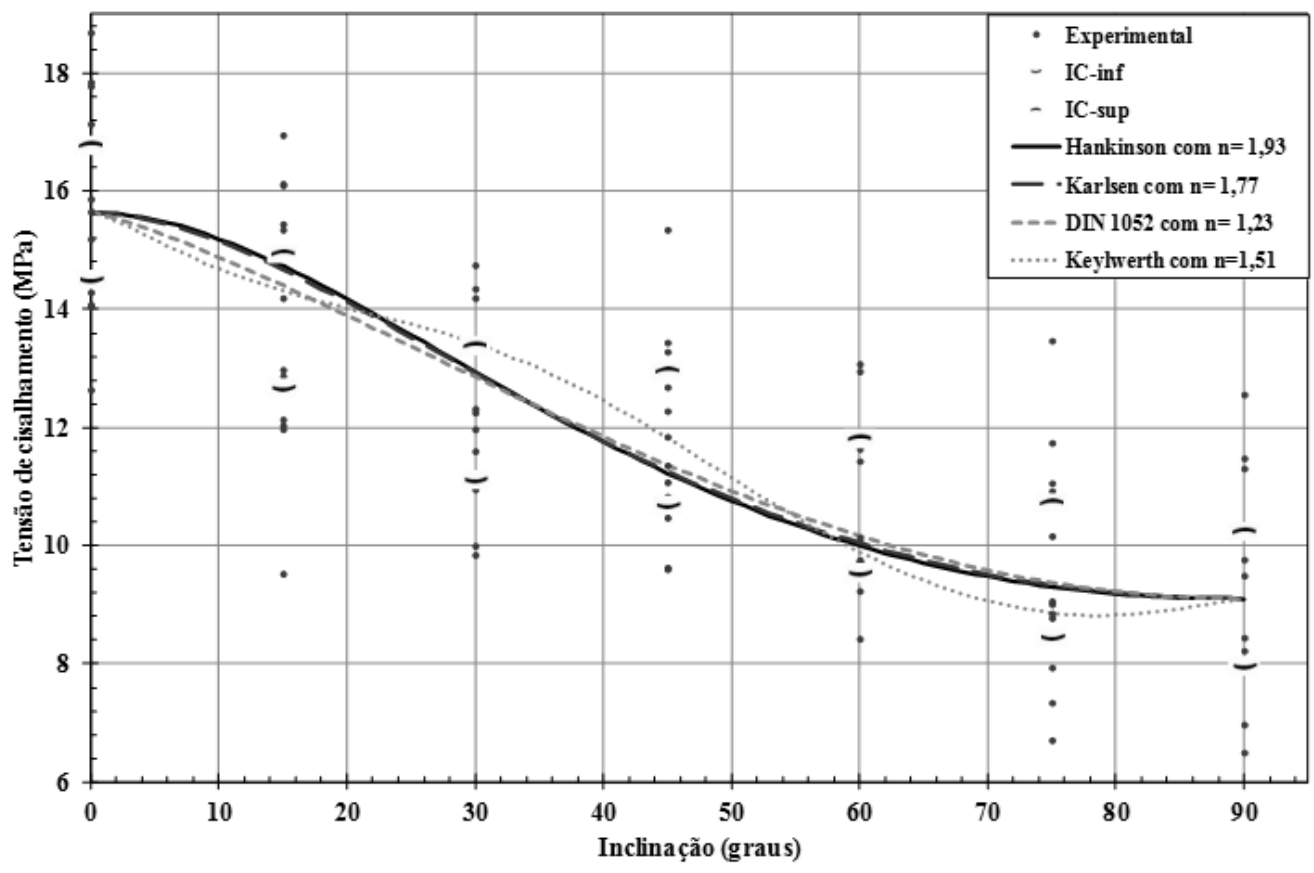

FIGURA 5: Tensão de cisalhamento x inclinação das fibras. Equações com expoente " $n$ " que deu maior significância estatística.

FIGURE 5: Shear stress versus inclination of the fibers. Equations with exponent " $n$ " which gave greater statistical significance. 
da inclinação das fibras, de todas as equações, dentro do intervalo de confiança das tensões de cisalhamento experimentais (nível de significância de $95 \%$ ), elaborou-se a representação gráfica das tensões de cisalhamento estimadas pelas equações, utilizando o expoente " $n$ " que resultou em uma maior significância estatística, e as experimentais, Figura 5.

Da análise dos gráficos da Figura $6 \mathrm{e}$ levando em consideração as análises estatísticas, pode-se afirmar que, em ordem de significância, as equações que melhor representam os valores experimentais são: DIN 1052 (equação 6), Karlsen (equação 7) e Hankinson (equação 8).

$$
\begin{gathered}
f_{v, \theta}=f_{v, 0}-\left(f_{v, 0}-f_{v, 90}\right) \times \sin (\theta)^{1,23} \\
f_{v, \theta}=\frac{f_{v, 0}}{1+\left(\frac{f_{v, 0}}{f_{v, 90}}+1\right) \times \sin (\theta)^{1,77}} \\
f_{v, \theta}=\frac{f_{v, 0} \times f_{v, 90}}{f_{v, 0} \times \sin (\theta)^{1,93}+f_{v, 90} \times \cos (\theta)^{1,93}}
\end{gathered}
$$

\section{CONCLUSÃO}

Dos quatro modelos matemáticos avaliados, os modelos de Karlsen e Keylwerth não apresentam significância estatística em seu formato original. Com as modificações feitas nos modelos, os que apresentam melhor ajuste foram, em ordem de significância: DIN 1052, Karlsen e Hankinson. Quaisquer desses três modelos matemáticos representam, satisfatoriamente, os resultados experimentais.

\section{AGRADECIMENTOS}

À FAPEMIG pelo auxílio financeiro.

\section{REFERÊNCIAS BIBLIOGRÁFICAS}

ASSOCIAÇÃO BRASILEIRA DE NORMAS TÉCNICAS. NBR 7190: Projeto de estruturas de madeira, Rio de Janeiro 1979.

AMERICAN SOCIETY FOR TESTING AND MATERIALS. Standard methods of testing small clear specimens of timber. ASTM D-143-94. Annual book of ASTM Standards, 2007.

BODIG, J.; JAYNE, B. A. Mechanics of wood and wood composites. 2. ed. Florida: Krieger publishing company, $1982.712 \mathrm{p}$.

DAHL, K. B.; MALO, K. A. Nonlinear shear properties of spruce softwood: experimental results. Wood Science and Technology, Berlin, v. 43, p. 539-558, 2009.

DINWOODIE, J. M. Timber: a review of the structure-mechanical property relationship. Journal of Microscopy, Oxford, v. 104, p. 3-32, 1975.

DUMAIL, J. F.; OLOFSSON, K.; SALME, N. L. An analysis of rolling shear of spruce wood by the Iosipescu method. Holzforschung, Berlin, v. 54, p. 420- 426, 2000.

EUROPEAN COMMITTEE FOR STANDARDI-

ZATION. Eurocode 5 - Design of timber structures - Part 1-1: General - Common rules and rules for buildings, 2005.

FOREST PRODUCTS LABORATORY. Wood handbook: wood as an engineering material. Gen. Tech. Rep. FPL-GTR-113. United States Department of Agriculture, Forest Products Laboratory, 1999.

GREKIN, M.; SURINI, T. Shear strength and perpendicular-to-grain tensile strength of defectfree Scots pine wood from mature stands in Finland and Sweden. Wood Science and Technology, Berlin, v. 42, p. 75-91, 2008.

HASSEL, B. I. et al. The single cube apparatus for shear testing - Full-field strain data and finite element analysis of wood in transverse shear. Composites Science and Technology, Elsevier, v. 69, p. 877-882, 2009.

KARLSEN, G. G. et al. Wooden Structures. 1. ed. Moscow: Mir Publishers, 1967. 638 p.

KOLLMANN, F. F. P.; CÔTÉ, W. A. Principles of wood science and technology: I Solid wood. 1. ed. Berlin: Springer, 1984. 592 p.

LIU, J. Y. et al. An improved shear test fixture using the Iosipescu specimen. Mechanics Cellul Materials, v. 231, n. 85, p. 139-147, 1999.

New shear strength test for solid wood.

Wood and Fiber Science, Hanover, v. 16, n. 4, p. 567-574, 1984.

LIU, J. Y.; ROSS, R. J. Wood property variation with grain slope. In: 12TH ENGINEERING MECHANICS CONFERENCE, 17-20 May 1998. American Society of Civil Engineers. Anais... La Jolla, CA, USA, 1998.

LIU, J. Y.; ROSS, R. J.; RAMMER, D. R. Improved Arcan shear test for wood. In: INTERNATIONAL WOOD ENGINEERING CONFERENCE, 28-31 October 1996. Anais... New Orleans, LA, USA, 1996.

LOGSDON, N. B. Variação da tensão resistente 
ao cisalhamento paralelo às fibras em uma peça de dimensões comerciais de AMESCLA, Trattinickia burserifolia (Mart.) Wild. In: ENCONTRO BRASILEIRO EM MADEIRAS E EM ESTRUTURAS DE MADEIRA, 5. 1995, Belo Horizonte, Anais... Belo Horizonte: EEUFMG; CEFET; IBRAMEM, 1995. v. 2, p. 235-244.

LOGSDON, N. B.; JESUS, J. M. H. Influência do ângulo entre o esforço aplicado e a direção das fibras da madeira sobre a resistência ao cisalhamento. Revista Engenharia Civil, Braga, v. 37, p. 5-16, 2010.

OLIVEIRA, J. M. Q. Characterization of the shear behaviour of wood using the Arcan test. 2004. 164 f. Dissertação (Mestrado em Engenharia Civil) - Universidade de Trás-os-Montes e Alto Douro, Portugal, 2004.

SZÜCS, C. A. Estudo do comportamento da madeira a esforços inclinados, In: ENCONTRO BRASILEIRO EM MADEIRAS E EM ESTRUTURAS DE MADEIRA, 4., 1992, São Carlos. Anais... São Carlos: EESC-USP; IBRAMEM. 1992. v. 2, p. 53-60.

UKYO, S.; MASUDA, M. A new method for measuring the true shear strength of wood. In: WORLD CONFERENCE ON TIMBER
ENGINEERING，9., 2006, Portland, Anais... Portland, v. 3, p. 41-47.

WANGAARD, F. F. The mechanical properties of wood. 1. ed. New York: John Wiley \& Sons, 1950. $377 \mathrm{p}$.

YAMASAKI, M.; SASAKI, Y. Elastic properties of wood with rectangular cross section under combined static axial force and torque. Journal of Materials Science, Springer, v. 38, p. 603-612, 2003. Yield behavior of wood under combined static axial force and torque. Experimental Mechanics, Springer, v. 44, n. 3, p. 221-227, 2004.

YOSHIHARA, H. et al. Comparisons of shear stress/ shear strain relations of wood obtained by Iosipescu and torsion tests. Wood and Fiber Science, Hanover, v. 33, n. 2, p. 275-283, 2001.

YOSHIHARA, H.; FURUSHIMA, T. Shear strengths of wood measured by various short beam shear test methods. Wood Science and Technology, Berlin, v. 37, p. 189-197, 2003.

YOSHIHARA, H.; MATSUMOTO, A. Measurement of the shearing properties of wood by in-plane shear test using a thin specimen. Wood Science and Technology, Berlin, v. 39, p. 141-153, 2005. 